\title{
BMJ Open Early point-of-care focused echocardiographic asystole as a predictive factor for absence of return of spontaneous circulatory in out-of- hospital cardiac arrests: a study protocol for a prospective, multicentre observational study
}

\author{
François Javaudin, ${ }^{1,2}$ Philippe Pes, ${ }^{2}$ Emmanuel Montassier, ${ }^{1,2}$ Arnaud Legrand, ${ }^{3}$ \\ Aline Ordureau, ${ }^{3}$ Christelle Volteau, ${ }^{3}$ Idriss Arnaudet, ${ }^{2}$ Philippe Le Conte ${ }^{1,2}$
}

To cite: Javaudin F, Pes $P$, Montassier E, et al. Early point-of-care focused echocardiographic asystole as a predictive factor for absence of return of spontaneous circulatory in out-of-hospital cardiac arrests: a study protocol for a prospective, multicentre observational study. BMJ Open 2019;9:e027448. doi:10.1136/ bmjopen-2018-027448

- Prepublication history and additional material for this paper are available online. To view these files, please visit the journal online (http://dx.doi. org/10.1136bmjopen-2018027448).

Received 23 October 2018 Revised 10 July 2019 Accepted 22 July 2019

Check for updates

(c) Author(s) (or their employer(s)) 2019. Re-use permitted under CC BY-NC. No commercial re-use. See rights and permissions. Published by BMJ.

For numbered affiliations see end of article.

Correspondence to

Professor Philippe Le Conte; philippe.leconte@chu-nantes.fr

\section{ABSTRACT}

Introduction Management of out-of-hospital cardiac arrests (OHCAs) in France is performed by a particular prehospital system based on medicalisation of mobile intensive care units composed of an emergency physician and a nurse with all the required devices for advanced care. It follows the European recommendations which advocate for the use of early point-of-care focused echocardiography (EPOCE) in the prehospital setting. An ability of EPOCE may be to predict the absence of return of spontaneous circulation (ROSC) in cases of absence of cardiac motion. We thus intended to investigate this predictive value with a prospective multicentre study. This paper describes the study protocol, while the first patients were recruited in December 2018.

Methods ACE is a prospective multicentre $(n=8)$ prognostic study. Briefly, as soon as OHCA is diagnosed and advanced life support (ALS) is initiated, EPOCE will be performed during the automated external defibrillator' analysis period. The physician will assess detectable motion within the heart and reversible causes of OHCA. However, as the prognostic value of absence of cardiac motion is not currently validated, the results of EPOCE will not be used to withdraw ALS, and the decision to withdraw life support will be done following the European Resuscitation Council recommendations during our study.

Analysis The primary endpoint is the positive predictive value of absence of cardiac motion for the absence of final ROSC. The secondary endpoints are predictive characteristics of EPOCE asystole on morbimortality 30 days after OHCA, description of reversible cause and analysis of the EPOCE technique.

Ethics and dissemination ACE was approved by an ethical committee (2018-A01491-54). While ACE is adapted to the French prehospital system, its results will be translatable to other organisations if inter-rater variability is not found.
Strengths and limitations of this study

- The study has a broad inclusion criteria, which would allow extrapolation to rather all out-of-hospital cardiac arrests.

The study plans to recruit a high number of patients.

- A previous pilot study has demonstrated the feasibility of this protocol.

- Cardiac massage interruption will be monitored in three out of eight centres.

This is an observational not an interventional study.

Trial registration number NCT03494153.

\section{INTRODUCTION}

Out-of-hospital cardiac arrests (OHCAs) are a major cause of mortality in France (between 30000 and 50000 cases per year). ${ }^{12}$ The prognosis is particularly poor, since only $5 \%-6 \%$ of patients will leave the hospital alive with satisfying neurological condition. ${ }^{2-4}$ Their management in France is performed by a particular prehospital system based on medicalisation of both ambulance dispatch (SAMU) and mobile intensive care units (SMUR), and follows the European recommendations (cardiac massage, ventilation, cardiac rhythm analysis, drug administration and defibrillation if needed). ${ }^{15}$ Based on published studies, ${ }^{6-8}$ the 2015 European recommendations advocate for the use of point-of-care focused echocardiography (POCE) in the prehospital setting, in particular to identify reversible causes of OHCA. ${ }^{16}$ Indeed, POCE can reveal various reversible 
causes such as tamponade, massive pulmonary embolism, deep hypovolaemia or suffocating pneumothorax. ${ }^{67}$ Their identification allows the clinician to better adjust his therapeutic strategy and accordingly might improve the patient's prognosis.

Another ability of POCE is to predict the absence of return of spontaneous circulation (ROSC) at the end of an advanced life support (ALS) procedure in cases of absence of cardiac motion. ROSC was defined as a spontaneous cardiac rhythm accompanied by breathing, coughing, movements or fleeting palpated pulse, according to the recommendations of Utstein registries. ${ }^{9}$ Several studies found a strong correlation between absence of cardiac motion and absence of ROSC. ${ }^{6-810} 11$ This fact deeply impacts extracorporeal circulation indications and organ donation procedures. However, these studies, mainly performed in a hospital setting, included rather small populations and used different cardiac motion definitions and procedures. Furthermore, they cannot be extrapolated to OHCA because of differences in terms of delay, management and environment. In this context, the European Resuscitation Council (ERC) stated in 2015 that, while absence of cardiac motion is highly predictive of death, sensitivity and specificity have not been reported. Thus, usage of this ascertainment to determine premature termination of resuscitation is currently not recommended until publication of a pivotal study.

\section{METHODS AND ANALYSIS Objectives}

The ACE French national trial fits precisely into this bibliographic gap, the uncertainty on the diagnostic value of absence of cardiac motion for the absence of final ROSC. Our objectives are multiple:

- The main objective is to assess the positive predictive value (PPV) of early point-of-care focused echocardiography (EPOCE) asystole for the absence of final ROSC.

- The secondary objectives are the following:

1. Assess the prognostic value of EPOCE asystole (ie, within the first $12 \mathrm{~min}$ of ALS initiation) on survival at hospital admission and on morbimortality at day 30 after OHCA (D30).

2. Assess the prognostic performance of EPOCE asystole for the absence of final ROSC.

3. Assess the prognostic value of EPOCE asystole according to timing of initiation after ALS initiation (by $2 \mathrm{~min}$ increment).

4. Assess the relationship between EPOCE findings and ECG rhythms.

5. Describe the frequency and typology of reversible causes (tamponade, massive pulmonary embolism, deep hypovolaemia or suffocating pneumothorax) in the context of OHCA.

6. Describe the characteristics of EPOCE: timing and quality assessed by the operator and by an expert committee.
7. Assess the prognostic performance of EPOCE in patients with ventricular tachycardia.

8. Create a multifactorial score with EPOCE combined with other clinical parameters (composite prognostic tool combining myocardial and/or electrical activity, capnography, no/low flow duration, and clinical profile including sex and age) for the absence of final ROSC.

9. Measure the cardiac massage interruption associated with EPOCE realisation by video recording in three centres (Nantes, La Roche-sur-Yon and Bobigny).

\section{Design}

ACE was designed as a prospective, multicentre prognostic study. It is based on a rigorous methodology (prospective observational study with a unique protocol), has a high proof-level design and will recruit a large sample of patients $(n=624)$. The Standard Protocol Items: Recommendations for Interventional Trials checklist is shown in the online supplementary file. Recruiting centres include both rural and urban community and university hospitals. This pragmatic approach intends to validate the performance of EPOCE in the prediction of the absence of a final ROSC in cases of absence of cardiac motion in the out-of-hospital setting. If this hypothesis is validated, it will allow for shorter delays before extracorporeal membrane oxygenation (ECMO) or organ donation processes.

Echographic or EPOCE asystole is defined by the complete absence of cardiac motion (coordinated or fibrillation) and the absence of valve movements.

\section{Methods}

Patients

The inclusion criteria were all patients $>18$ years old presenting with an OHCA for whom an EPOCE has been initiated in less than $12 \mathrm{~min}$ after ALS initiation.

Non-inclusion criteria include a do not resuscitate order, ROSC prior to EPOCE, ALS not performed by the prehospital team, pregnancy, breastfeeding women and inmates.

\section{Procedures}

After verification of inclusion and exclusion criteria, ALS intervention will replace basic life support with an overlap period, as usual (figure 1). ALS will be performed according to the latest ERC regulations, including realisation of an ECG. Once standard ALS interventions are done according to the focused echocardiographic evaluation in life support (FEEL) protocol and ERC recommendations. ${ }^{45}$ The echography will be performed. The FEEL protocol was designed and evaluated in a prospective observational study using an ALS-compliant focused echocardiography. Briefly, once on the scene, if the patient was in cardiac arrest, cardiopulmonary resuscitation was started, an ECG was performed and a clinical diagnosis was established. A focused echocardiography was then performed. Outcome, defined as survival to admission, was better when cardiac motion was present regardless of 


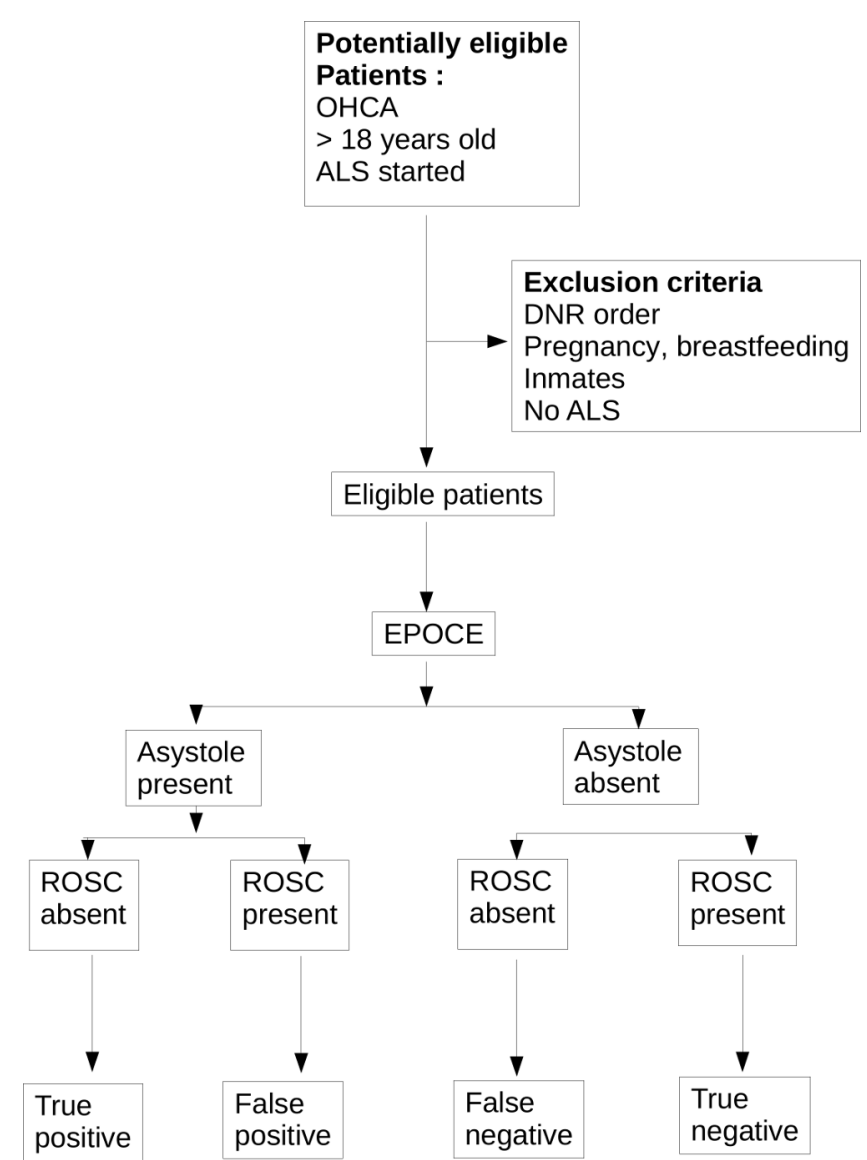

Figure 1 Patient's flow chart in the ACE trial. ALS, advanced life support; DNR, do not resuscitate; EPOCE, early point-of-care focused echocardiography; OHCA, outof-hospital cardiac arrest; ROSC, return of spontaneous circulation.

the initial rhythm. In our study, the physician will perform EPOCE during the defibrillator's analysis period, thus in less than $10 \mathrm{~s}$. It will be done using a phased array probe with a subcostal view. It has to be done as early as possible and always before $12 \mathrm{~min}$ after ALS initiation. The physician will observe for cardiac motion or lack thereof and will look for reversible causes. Video clips will be stored in the echographic device and secondarily uploaded in the electronic case report file (eCRF) for random review by an expert committee. The entire ALS procedure will be closely monitored to assess for diagnostic and therapeutic delays (defined as the interval between arrival time on the scene and therapeutic initiations). ALS will be terminated following the ERC rules, and the results of EPOCE will not be used for that purpose. For the study purpose, the presence or absence of ROSC will be assessed after ALS termination. In the case of ROSC, the hospital course (intensive care unit, medicine ward) will be described. At D30, the vital status of all patients will be assessed by either a hospital file consult or a phone call if the patient is still alive. For these patients, autonomy will be assessed using the Glasgow Outcome Scale. These two events (dead or alive and Glasgow Outcome Scale score) define morbimortality. They will be assessed by the research team of Nantes Hospital. In three centres, the entire resuscitation procedure will be monitored via a mobile video recorder. Video clips will be uploaded and analysed to measure the duration of cardiac massage interruptions.

\section{Endpoints}

Primary endpoint

- Predictive prognostic value of EPOCE asystole (ie, within the first $12 \mathrm{~min}$ of ALS initiation) on resuscitation failure (absence of ROSC). We have chosen PPV as the primary endpoint because we want to isolate a population without ROSC with EPOCE asystole.

\section{Secondary endpoints}

- Predictive prognostic value of EPOCE asystole (ie, within the first $12 \mathrm{~min}$ of ALS initiation) on hospital admission and on morbimortality (defined as dead or alive and Glasgow Outcome Scale score) evaluated at 30 days.

- Sensitivity, specificity and negative predictive value (NPV) of EPOCE asystole for the absence of final ROSC.

- Sensitivity, specificity, PPV and NPV of EPOCE asystole for the absence of final ROSC according to their timing of initiation after ALS initiation (by $2 \mathrm{~min}$ ).

- Association between the ultrasound asystole rate according to the cardiac electrical activity (pulseless activity, asystole, ventricular fibrillation and ventricular tachycardia).

- Description of reversible causes (tamponade, massive pulmonary embolism, deep hypovolaemia or suffocating pneumothorax), diagnostic (time between ALS onset and diagnosis) and therapeutic delays (time between ALS onset and specific therapeutic intervention), and the effectiveness of implemented curative strategies defined by the association with ROSC and 30-day morbimortality.

- Analysis of the EPOCE technique during OHCA resuscitation: duration, whole quality of the video clips assessed by the operator on a predetermined scale (from $0=$ impossible to $10=$ excellent) and an expert committee reviewing a $10 \%$ random sample.

- Sensitivity, specificity, PPV and NPV of EPOCE asystole to predict ROSC absence in the subgroup of patients with ventricular fibrillation on the ECG.

- Main determinants of death (age, sex, comorbidities, suspected aetiology, no/low flow duration, initial treatment, electrical activity and cardiac motion) associated with the absence of ROSC to determine a score with $100 \%$ PPV.

- Measure of duration of cardiac massage interruption in seconds during EPOCE, using a portable video recorder (three centres: Nantes, La Roche-sur-Yon and Bobigny). 


\section{Recruiting centres}

The recruiting centres will be university hospitals (Nantes, Brest, Tours, Angers and Bobigny) and community hospitals (Saint-Nazaire, La Roche-sur-Yon and Chateaubriant).

\section{Sample size calculation}

The principal objective is the PPV of absence of cardiac motion (asystole) for the absence of final ROSC. To specify the width of the CI at $\pm 3 \%$ with $95 \%$ PPV, 203 patients without cardiac motion are required. Based on $37.5 \%$ ultrasound asystole rate, ${ }^{10}$ a total of 542 patients are required. Taking into account $\mathrm{a}+15 \%$ attrition rate (incomplete data, too poor quality of the ultrasound for interpretation and so on), the required population will finally be 624 patients.

\section{Recruitment}

Chosen prehospital teams were recruited because they are highly skilled in clinical ultrasound and already use this technique in their emergency department. A monthly newsletter will be published with individual and global recruitment trends. Patients will be followed up until D30, and defined gradually as survival without neurological deficit, survival with neurological deficit or death.

\section{Data management}

eCRFs will be used via a web-based interface and video clips will be uploaded. All data will be stored in Nantes University Hospital's secured databases. The data management team will be responsible for the entire process. Data will be anonymised with an incremental number assigned to each patient. The final database will be available only to the steering committee. Subjects with missing data for the primary endpoint will not be analysed $(+15 \%$ subjects in sample size calculation).

\section{Monitoring}

Monitoring will be performed both by electronic surveillance of recruitment and data quality. It will be done by the Clinical Research Department of Nantes University Hospital.

\section{Statistical analysis}

Sensibility, specificity, PPV, NPV and likelihood ratio of EPOCE asystole on resuscitation failure (absence of ROSC) and on morbimortality will be estimated with 95\% CI. Logistic model regression and receiver operating characteristic curve will be estimated to analyse the time of EPOCE initiation that allows the best prognostic performance for the absence of final ROSC. $\chi^{2}$ and Fisher's test will be used to analyse the association between ultrasound diagnosis and ECG electrical patterns. Reversible causes, diagnostic and therapeutic delays, and the effectiveness of implemented curative strategies will be described. The EPOCE technique during the OHCA resuscitation and the duration and quality of the video clips will be described. $\chi^{2}$ and Student's t-tests will be used to test the association between the quality and duration of the videos. Prognostic performance of EPOCE to predict
ROSC absence in patients with ventricular fibrillation without cardiac motion will be estimated. A multifactorial composite prognostic score associated with the absence of ROSC will be constructed with a logistic regression model. Parameters that will be taken into account will be myocardial and/or electrical activity, capnography, no/ low flow duration, and clinical profile including sex and age. Measure of mean duration of cardiac massage interruption during EPOCE will be estimated. $\mathrm{P}$ values less than 0.05 will be considered statistically significant. All analyses will be performed using SAS V.9.4.

\section{ETHICS AND DISSEMINATION}

In accordance with the recommendations of Comité de Protection des Personnes 'Ile de France II' France, patient and/or legal authority consent will be requested only for survivors. Furthermore, with regard to the inclusion criteria, it will be impossible to seek for relatives' consent. With regard to the very low survival rate of patients with OHCA (5\%), anonymised database and family-induced traumatism, we have asked for a derogation to surrogate information for deceased patients. Consent will be requested for surviving patients. We intend to publish ACE results in a major journal of emergency medicine, and raw data will be available on reasonable request.

\section{PATIENT AND PUBLIC INVOLVEMENT}

Patients and the public had no involvement in the design or planning of the study.

\section{DISCUSSION}

There is a strong rationale on the interest on early diagnosis of ROSC absence in OHCA. It might allow premature initiation of ECMO indications or organ donation procedures without waiting for a median time of $30 \mathrm{~min}$ after ALS onset. Conversely, in cases of cardiac motion visualisation, a far better prognosis is likely and prehospital teams might search for reversible causes.

Hard evidence is currently missing in the literature since the majority of published studies included small series of patients, in the hospital, and used different protocols. $^{6-810}$ A multicentre study was performed, but EPOCE was performed in the emergency department even if the cardiac arrest occurred out of hospital. ${ }^{12}$ This was stated by the ERC in its 2015 recommendations. ${ }^{1} \mathrm{ACE}$ is adapted to the particularities of the French prehospital system, but its results will be translatable to other organisations such as European or American organisations.

ACE has the potential to provide a definitive response to this question. Furthermore, it will answer the question on the frequency of reversible causes and their management, which also needs hard evidence. ACE has the potential to provide this evidence since it will include 624 non-selected patients with OHCA. 
POCE has been associated with delays in chest compressions ${ }^{13}$ which would alter prognosis. However, the training of physicians in POCE in this previous study was not reported. ${ }^{13} 14$ The study was performed in the USA, and it might be assumed that the training was in line with established residency training requirements. This potential flaw has to be addressed; in ACE, physicians will be trained before the trial's onset, and cardiac massage interruption will be recorded and measured in a subgroup of patients.

\section{Author affiliations}

${ }^{1}$ Emergency Medicine, Université de Nantes Faculte de Médecine, Nantes, France

${ }^{2}$ Emergency Department, Centre Hospitalier Universitaire de Nantes, Nantes, France

${ }^{3}$ Centre Hospitalier Universitaire de Nantes, Nantes, France

Contributors FJ, PP, IA and PLC conceived and wrote the protocol. AL and AO brought methodological and administrative help. CV was in charge of the statistical aspect. EM reviewed the whole process.

Funding ACE is supported by a $€ 193000$ grant from the French Ministry of Health (PHRC-IR 2017) (grant number API17/N/035). ACE is currently supported by the French Society of Emergency Medicine (Société Française de Médecine d'Urgence) and by Winfocus France without funding. There is ongoing discussions with Philips and SonoSite for the loan of echographic devices to increase the number of available devices for prehospital teams. Funders have no role in the ACE study.

Competing interests None declared.

Patient consent for publication Not required.

Ethics approval The ACE trial has been approved by the ethics committee (Comité de Protection des Personnes 'Ile de France II' France, 2018-A01491-54).

Provenance and peer review Not commissioned; externally peer reviewed.

Open access This is an open access article distributed in accordance with the Creative Commons Attribution Non Commercial (CC BY-NC 4.0) license, which permits others to distribute, remix, adapt, build upon this work non-commercially, and license their derivative works on different terms, provided the original work is properly cited, appropriate credit is given, any changes made indicated, and the use is non-commercial. See: http:// creativecommons.org/licenses/by-nc/4.0/.

\section{REFERENCES}

1. Soar J, Nolan JP, Böttiger BW, et al. European resuscitation Council guidelines for resuscitation 2015: section 3. adult advanced life support. Resuscitation 2015;95:100-47.

2. Luc G, Baert V, Escutnaire J, et al. Epidemiology of out-of-hospital cardiac arrest: a French national incidence and mid-term survival rate study. Anaesth Crit Care Pain Med 2019;38.

3. Nicolas G, Lecomte D. [Sudden cardiac death in adults. Epidemiology]. Bull Acad Natl Med 1999;183:1573-9.

4. Les statistiques publiques | RéAC, 2019. Les statistiques publiques. Available: http://registreac.org/?page_id=2822 [Accessed 2019, August 20].

5. Stiell IG, Nichol G, Leroux BG, et al. Early versus later rhythm analysis in patients with out-of-hospital cardiac arrest. N Engl J Med 2011;365:787-97.

6. Breitkreutz R, Price S, Steiger HV, et al. Focused echocardiographic evaluation in life support and peri-resuscitation of emergency patients: a prospective trial. Resuscitation 2010;81:1527-33.

7. Blyth L, Atkinson P, Gadd K, et al. Bedside focused echocardiography as predictor of survival in cardiac arrest patients: a systematic review. Acad Emerg Med 2012;19:1119-26.

8. Tsou P-Y, Kurbedin J, Chen Y-S, et al. Accuracy of point-of-care focused echocardiography in predicting outcome of resuscitation in cardiac arrest patients: a systematic review and meta-analysis. Resuscitation 2017:114:92-9.

9. Jacobs I, Nadkarni V, Bahr J, et al. Cardiac arrest and cardiopulmonary resuscitation outcome reports: update and simplification of the Utstein templates for resuscitation registries: a statement for healthcare professionals from a task force of the International liaison Committee on Resuscitation (American Heart Association, European Resuscitation Council, Australian Resuscitation Council, New Zealand Resuscitation Council, Heart and Stroke Foundation of Canada, InterAmerican Heart Foundation, Resuscitation Councils of Southern Africa). Circulation 2004;110:3385-97.

10. Aichinger G, Zechner PM, Prause G, et al. Cardiac movement identified on prehospital echocardiography predicts outcome in cardiac arrest patients. Prehosp Emerg Care 2012;16:251-5.

11. Kim HB, Suh JY, Choi JH, et al. Can serial focussed echocardiographic evaluation in life support (FEEL) predict resuscitation outcome or termination of resuscitation (TOR)? A pilot study. Resuscitation 2016;101:21-6.

12. Gaspari R, Weekes A, Adhikari S, et al. Emergency department point-of-care ultrasound in out-of-hospital and in-ED cardiac arrest. Resuscitation 2016;109:33-9.

13. Huis In 't Veld MA, Allison MG, Bostick DS, et al. Ultrasound use during cardiopulmonary resuscitation is associated with delays in chest compressions. Resuscitation 2017;119:95-8.

14. Lapostolle F, Le Conte P, Arnaudet I, et al. Point-of-care ultrasound during advanced cardiopulmonary resuscitation: rule of art has to be respected! Resuscitation 2018;122:e1. 